\title{
Gene action in the mammalian $\mathrm{X}$-chromosome
}

\author{
BY HANS GRÜNEBERG \\ Department of Animal Genetics, University College London
}

(Received 16 November 1966)

\section{INTRODUCTION}

So much has been written about the Lyon Hypothesis in recent years that it is scarcely necessary to re-state it here in detail. Suffice it to say that it postulates the random and irreversible inactivation of one of the two $\mathrm{X}$-chromosomes during embryonic development in the mammalian female, with the result that in any individual cell, a single $\mathrm{X}$-chromosome only is active as in the XY male. The evidence for the Lyon Hypothesis (or L.H. for short) is partly cytological and partly genetical. This paper is not concerned with its cytological aspects. The genetical evidence came, in the first instance, from the peculiar mottled or mosaic phenotype of certain heterozygotes for sex-linked genes (such as the tortoiseshell cat) which suggested to Lyon $(1961,1962)$ that the contrasted patches were descended from cells in which either the $\mathrm{X}$-chromosome carrying one or that carrying the other allele had been inactivated.

Until very recently, little was known as to whether heterozygotes for individual sex-linked genes carried in structurally normal $\mathrm{X}$-chromosomes do in fact behave according to the L.H. Such information is essential for the critical evaluation of more complex situations (such as the simultaneous segregation of two sex-linked genes, or the behaviour of genes in $\mathrm{X}$-autosome translocations) which have been studied by Lyon and several other investigators.

The sex-linked gene tabby $(T a)$ in the mouse, in addition to its known effects on skin and coat, also produces a characteristic dental syndrome (Grüneberg, 1965). $T a /+q 9$ often show a spectacular mixture of normal, tabby-type and mixed molars which at first was regarded as strong prima facie evidence in favour of the L.H. However, a detailed study (Grüneberg, 1966a) showed that the behaviour of the $T a /+$ molars could not be accounted for in terms of the L.H. The main discrepancies are that the involvement of the various teeth is not random, but follows a definite pattern, that the contribution of tabby and tabby-like molars is far less than onehalf as expected on the L.H., and that an autosomal mimic of tabby, crinkled (cr), shows an essentially similar behaviour of its teeth in $+/ c r$ heterozygotes. This led to a re-investigation of the other effects of tabby (Grüneberg, 1966b) with the result that in every single feature, the behaviour of tabby was at variance with the consequences of the L.H. Nor is tabby unique in this respect. Other sex-linked genes in the mouse (striated, Str; brindled, $M o^{b r}$ and bent-tail, $B n$ ) disagree with the L.H. 
wherever evidence is available; in particular, it has been shown that in Str/+ and $M o^{b r} /+$ o $ᄋ$ (and probably in $T a /+q O+$ as well), the fur as a whole is abnormal, and not only the short-haired, light-coloured etc. stripes and regions by which the mutants were identified in the first instance. Other sex-linked heterozygotes in guinea pig, cat and cattle tell the same story. In man (Grüneberg, 1967), none of the sex-linked genes with 'visible' heterozygous manifestation presents as much as a plausible prima facie case in favour of the L.H. In four cases, the gene effect has been traced to the cellular level; in one of these there is no evidence for the existence of two cell populations such as would be required by the L.H. In the other three instances, there is evidence for gene inactivation at the cellular level, but the 'mosaic', if it exists in vivo, is so fine-grained as to be difficult to reconcile with the L.H. which postulates inactivation early in embryonic development. Moreover, there is evidence that the phenomenon occurs both in sex-linked and in autosomal conditions.

In the light of this new evidence it becomes necessary to re-assess the remaining and more complex genetic situations which have been thought to support the L.H. It will be shown that these data, so far from supporting the L.H., create further difficulties for it: in particular, their interpretation in terms of the L.H. reveals internal contradictions which appear to be irreconcilable. An alternative hypothesis is therefore put forward which postulates that in the female of the mouse, and presumably of other mammals, genes on both X-chromosomes are physiologically active, but only at about half-strength. The hypothesis is in agreement with all genetical data and, like the L.H., it provides a mechanism to account for dosage compensation of sex-linked genes.

\section{DOUBLE HETEROZYGOTES FOR SEX-LINKED GENES}

According to the L.H., two sex-linked genes $A$ and $B$ acting through the same cells, in the repulsion phase $(A+1+B)$, should manifest themselves in patches which are phenotypically either $A$ or $B$, never both or neither. Conversely, in the coupling phase $(A B /++)$, the contrasted patches should be either $A$ and $B$, or their two normal alleles, but never $A$ or $B$ separately. As a corollary, interaction between $A$ and $B$ should be possible in the coupling phase (where both are simultaneously active in the same cells), but not in the repulsion phase (where, in any particular cell, either one or the other is active, but never both at the same time).

The use of double heterozygotes as a test for the validity of the L.H. (Lyon, 1963) is, of course, contingent on the fact that, in single heterozygotes, both $A$ and $B$ behave in conformity with the L.H.: i.e., that each gene individually forms patches of pure mutant and pure wild-type phenotype according to the criteria discussed elsewhere (Grüneberg, 1966b). As it has now been shown that, in single heterozygotes, the genes for tabby, striated and brindled etc. affect the coat as a whole and not only the stripes, short-haired areas and light-coloured patches respectively, the synthesis of double heterozygotes loses its point as a test for the validity of the L.H.: as the genes demonstrably act over the coat as a whole, the phenotype of 
two of them in double heterozygotes, no matter what it is, is the result of factor interaction and nothing else.

Interesting though they may be in other respects, the details of the interaction between these various genes may thus be disregarded in the present discussion. However, the existence of factor interaction in the repulsion phase, if it can be demonstrated, would be additional evidence for the action of both genes in the same cells and hence against the validity of the L.H. Needless to say, failure to demonstrate such interaction is no evidence in favour of the L.H. as, after all, not all genes interact with each other.

Double heterozygotes for tabby and striated $(T a+/+S t r)$ have been described by Lyon (1963). Those born in this laboratory also have naked tails and bare patches behind the ears and are in this respect like $T a{ }^{\hat{\sigma}} \hat{\sigma}$ or $T a / T a$ 우 and quite unlike $T a /+$ \&ᄋ․ Evidently, Str enhances the effect of $T a$ on these features so that the heterozygotes look like hemizygotes or homozygotes. Lyon's discussion of the coat suffers from the fact that the hair was examined under the dissecting microscope only, a method adequate for the enumeration of hair types in normal coats, but insufficient for coats which consist largely of atypical fibres. In particular, microscopic magnifications are required to distinguish between true constrictions (as in normal zigzags and auchenes) and angulations of hairs due to other reasons such as twisted-ribbon effects and the like. Details of the hair structure of various mouse mutants will be given in later publications; here a few short remarks may suffice. In the coat of tabby mice, as in that of crinkled ( $c r$; Falconer, Fraser \& King, 1951), none of the hairs has constrictions. In striated, the more extremely affected hairs are ribbon-like and twisted round their axis, with various abnormalities of the internal structure and often of pigmentation. There are no constrictions comparable to those in normal zigzags and auchenes. In the remainder of the coat, the same anomalies are found in a less extreme form; constrictions are present, but they seem to separate the segments less completely from each other than in normal hairs; thus unbroken sequences of septa are more often found throughout the length of the constricted regions. In $\mathrm{Ta}+1+S t r$ Oᄋ, most of the hairs lack constrictions altogether or have rudimentary constrictions. The fur is thus affected more strongly than by $T a$ or by Str acting alone, both of which, it will be remembered, affect the coat as a whole. The two genes thus reinforce each other in their effect on the coat. Otherwise, the coat combines features of both mutants. In some animals, it comes close to the tabby phenotype, in others the coat more closely resembles that of the Str $/+$ mouse, except for the absence or rarity of constrictions and, of course, the presence of bare patches behind the ears and the naked tail.

In $T a+1+S t r$ OP, the two genes thus strikingly interact with each other. Simultaneous action of both genes over the coat as a whole and the tail is contrary to the expectation on the L.H., but easily understood in terms of factor interaction.

Double heterozygotes involving tabby and dappled $\left(M o^{d p}\right)$ have been investigated by Lyon (1963). Here we shall deal with double heterozygotes of tabby and brindled $\left(M o^{b r}\right)$ as their coats have now been studied in greater detail microscopically. The situation encountered differs radically from that in the $T a+1+S t r$ qQ just 
described. Confining ourselves, in the first instance, to the underfur, the (middorsal) coat of $T a+1+M o^{b r}$ of consists almost entirely of two distinct kinds of fibres. Some of these are fully pigmented and of tabby type; i.e., they have irregular sequences of septa and septules, but no constrictions. The other fibres have little or no pigment, structural abnormalities typical of brindled, and they show normal constrictions; they are thus essentially of brindled type. Exceptions are so few in number and of such doubtful significance that I am not inclined to attach much importance to them. The overhairs cannot be fully classified, but so far as the evidence goes, it confirms that of the underfur: in particular, the strongest awls, with three to five rows of septules, are all unpigmented and hence of brindled type; in the coat of tabbies, awls never seem to have more than two rows of septules. In so far as the fibres of these repulsion heterozygotes can be classified, they thus consist of two types corresponding to tabby and brindled respectively.

The situation is quite different in the coupling phase (actually, the animals also carried the gene for sex-linked anaemia, sla, and their full constitution was thus $T a M o^{b r}+1++s l a$; it is believed that the presence of $s l a$ had no influence on the situation under discussion and it will thus be ignored). Much of the fur of these animals, but by no means the whole of it, again consists of two kinds of fibres. But this time, the predominating hair types are different: the underfur consists mainly of fully pigmented fibres with constrictions (i.e., corresponding to normal) and of fibres with little or no pigment, structural anomalies of various kinds and no constrictions (i.e., fibres combining features of both mutant genes). But in addition to these fibres which correspond in phenotype to ++ and $T a M o r r$ respectively, all five individuals whose coat has been studied have an appreciable number of fibres of different types. In the underfur, some unpigmented hairs have constrictions whereas others have unpigmented regions and constrictions; these hairs thus show, wholly or in part, the phenotype of $M o^{b r}$, but not that of $T a$. Similarly, some of the strongest awls are unpigmented and with structural anomalies typical of brindled: as there are no tabby awls of that calibre, it must be concluded that, as in the underhairs just described, $M o^{b r}$ but not $T a$ is manifest. By contrast, hairs clearly manifesting $T a$ but not $M o^{b r}$ have not been observed.

On first sight, the similarity with the consequences of the L.H. appears indeed striking. In the $T a+1+M o^{b r}$ mouse, virtually all the underhairs and probably all the hairs are either of the tabby or of the brindled phenotype; and in the $T a M o^{b r} /++$ mouse, the majority of the hairs are of the double-mutant or of the normal phenotype. However, in the coupling phase, numerous hairs are present which are brindled but not tabby. Moreover, the observations would only fit the L.H. on the assumption that, individually in single heterozygotes, $T a$ and $M o^{b r}$ behave according to the L.H. In actual fact, they do not and in particular, they affect the coat as a whole and not only parts of it. Nor is it legitimate to consider the $T a-M o^{b r}$ double heterozygotes in isolation. The $T a+1+S t r$ mouse clearly shows that the two genes interact with each other over the whole of the coat and the tail, in agreement with the overall action of the two genes individually. The tabby gene is common to both: the $T a+/+S t r$ phenotype shows that tabby is active all over the coat, the 
$T a+1+M o^{b r}$ and $T a M o^{b r} /++$ phenotypes show that it is active in some of the hair follicles, but not in others. For the L.H., this is an insoluble paradox. In terms of factor interaction, there is no such difficulty : there is nothing strange in the notion that the interaction of tabby with striated should be physiologically different from that with brindled.

We conclude that the behaviour of the double heterozygotes can be consistently accounted for in terms of factor interaction, but not in terms of the L.H. As a system for the investigation of single-gene effects and of factor interaction, mammalian hair has some considerable advantages. Unlike the macroscopic effects which are commonly studied, the individual hair is the product of a single hair follicle and hence of a comparatively small number of cells. It preserves, like the rings of a tree, a record of follicular activity in time, and hairs produced by the same follicle in successive hair growth cycles can be studied. The interesting cis-trans difference of $T a-M o^{b r}$ heterozygotes first described in this paper, genes separated from each other by some four centimorgans, is thus well worth a more detailed study.

\section{X-AUTOSOME TRANSLOCATIONS}

Two distinct but complementary types of evidence have been adduced in favour of the L.H. In one instance, a segment of the $\mathrm{X}$-chromosome, including several known loci, has been transferred to an autosome: this made it possible to study the behaviour of normally sex-linked genes when carried on an autosome. In a series of cases, segments of autosomes carrying known loci have been transferred to the $\mathrm{X}$-chromosome: this made it possible to study the behaviour of normally autosomal genes when carried on the X-chromosome.

\section{(i) Transfer of sex-linked genes to an autosome}

In a reciprocal translocation (variously known as Searle's translocation, T16H or Tx), a segment of the X-chromosome carrying several known loci has been transferred to a hitherto unidentified autosome (Lyon, Searle, Ford \& Ohno, 1964; Ohno \& Lyon, 1965). In each case, the manifestation of the gene is strikingly changed depending on its position in the genome. Whereas the ordinary $T a /+q$ is striped, a $\mathbf{T x}+1+T a$ o (with the normal allele in the translocated segment) is phenotypically normal; conversely, a $T \times T a /++q$ (with $T a$ in the translocated segment) has the phenotype of the tabby hemizygote or homozygote. The same happens to blotchy which, in an ordinary $B l o /+$ heterozygote, has a mottled or roan type of coat: $\mathrm{Tx}+/+B l o$ is phenotypically normal, whereas $\mathrm{Tx} B l o /++$ has the phenotype of the blotchy hemizygote or homozygote and is light all over (except for the head). For bent-tail, only $\mathbf{T x}+/+B n$ has so far been obtained: it is phenotypically normal whereas tail abnormalities are present in the majority of $B n /+$ qOOP. Quite recently (Lyon, 1966), it has also been shown that the gene for sparse-fur $(s p f)$ shows a similar behaviour; as judged by eye, the majority of ordinary $+/ s p f q q$ 
are phenotypically normal, but some are mildly affected though without showing mosaicism for this character. By contrast, Tx spf $/++$ of show the full sparse-fur phenotype. Though, in the absence of microscopic studies, the nature of the $+/ s p f$ heterozygote remains problematical, the behaviour of the gene when in the $\mathrm{Tx}$ chromosome follows the general pattern. To avoid a loaded terminology, it may be said that whichever allele of such a structural heterozygote is carried on the autosome is 'expressed' whereas the allele carried on the intact X-chromosome is not 'expressed'.

For tabby, the present author has extended these observations to the molars. All twenty-seven $\mathrm{Tx}+1+T a$ ㅇ examined had normal molars and were in this respect quite different from ordinary $T a /+$ q (Grüneberg, 1966a). By contrast, all six $\mathrm{Tx} T a /++$ + $Q$, with an exception to be mentioned below, had typical tabby-type molars, again quite different from ordinary $T a /+$ oㅇ. There was thus an (almost) complete correlation between type of fur and type of teeth.

Evidently, the expression of all these genes is subject to a position effect. But the facts in themselves reveal nothing about its nature. In particular, are the alleles carried on the intact $\mathrm{X}$-chromosome weakened or those on the autosome strengthened? Both statements are logically equivalent and equally valid descriptions of the facts. Hence, the data unaided cannot discriminate for or against the L.H. If we consider the evidence from Searle's translocation in isolation; and if, in a structurally normal $\mathrm{X}$-chromosome, the heterozygotes for the various genes behaved in accordance with the L.H. (as the authors presume), it would be legitimate to interpret their behaviour in the $\mathrm{T} 16 \mathrm{H}$ translocation in terms of a modification of that hypothesis (the genes in the translocated segment being regularly 'active' and their alleles in the intact $X$ being regularly 'inactive': i.e., chromosome inactivation, in this case, is not random, but always involves the intact X-chromosome). Conversely, if the genes, in a structurally normal Xchromosome, behave like ordinary autosomals, it is equally legitimate to interpret their behaviour in $\mathrm{T} 16 \mathrm{H}$ in terms of conventional dominance (the alleles in the translocated segment being 'dominant' and those on the intact $\mathrm{X}$-chromosome 'recessive'). Looked at in isolation, the behaviour of the genes in the $\mathrm{T} 16 \mathrm{H}$ translocation thus cannot be interpreted except in conjunction with that of the individual genes in structurally normal chromosomes. And as it has been shown (Grüneberg, $1966 b)$ that $T a, S t r, M o^{b r}$ and probably $B n$ behave like ordinary autosomal genes in heterozygotes (as is presumably true for Blo even if it should not turn out to be an allele of $M o$, and for $s p f$ ), there is no valid reason why these data should be interpreted in terms of the L.H. As will be shown in a later section, when the evidence from Searle's translocation is considered in conjunction with that from the other $\mathrm{X}$-autosome translocations, much firmer conclusions will emerge.

On the L.H., the phenotype of $\mathrm{Tx}+/+T a$ would have to be completely normal and that of $\mathrm{Tx} T a /++$ like tabby exactly. The conventional interpretation would also be compatible with incomplete dominance. I have failed to discover convincing evidence for incomplete dominance as regards the coat in either genotype (though such might come to light in a more detailed study), and the molars of the twenty- 
seven $\mathrm{Tx}+/+T a$ 워 seemed to be completely normal. But a somewhat suggestive feature was noticed in the molars of the six $\mathrm{Tx} T a /++$ oㅇ examined. In normal mice, the first and second upper molars $\left(\mathrm{m}^{1}\right.$ and $\left.\mathrm{m}^{2}\right)$ have three separate roots each. In tabbies, there is usually a single composite root only (Grüneberg, 1965, 1966a); this was so in $97 / 108 \mathrm{~m}^{1}$ whereas $11 / 108$ had the posterior root separate; and a single root was present in $108 / 108 \mathrm{~m}^{2}$. On the other hand, in $T a /+$ OP, molars with tabbytype crowns commonly have more or less separate roots. Now, in Tx $\mathrm{Ta} /++$ +o, $11 / 12 \mathrm{~m}^{1}$ and $8 / 12 \mathrm{~m}^{2}$ had separate posterior roots and in this respect resembled $T a /+$ 우 rather than $T a \hat{\delta} \delta^{\hat{0}}$ or $T a / T a$ 우. However, as no tabbies on the same genetical background are available, this evidence must be regarded as suggestive rather than critical.

(ii) Transfer of autosomal genes to the $X$-chromosome

Out of eight translocations between the $\mathrm{X}$-chromosome and an autosome hitherto described, seven are reciprocal and mostly derived from irradiation experiments at Oak Ridge, Tennessee (Russell \& Bangham, 1961 and earlier papers; Russell, Bangham \& Saylors, 1962; Russell, 1963, 1964; Russell, Bangham \& Montgomery, 1964; Russell \& Montgomery, 1965). One of these involves linkage group 8 (which includes the genes for brown $(b)$ and for misty $(m))$; the remainder involve linkage group 1 (which includes the albino locus $\left(c, c^{c h}\right.$, etc.), pink-eyed dilution $(p)$ and taupe $(t p)$ ). In addition there is Cattanach's translocation (Cattanach, 1961, 1966; Ohno \& Cattanach, 1962) which seems to be non-reciprocal and in which a segment of linkage group $\mathrm{l}$ is inserted into the continuity of the $\mathrm{X}$-chromosome.

All these translocations have been discovered through females with mottled or variegated coat colour. In the reciprocal translocations, these mottled females are heterozygous for a coat colour gene such that the normal allele is carried in the segment transferred to the $\mathrm{X}$-chromosome and the mutant allele on the intact autosome. In the case of Cattanach's translocation, mottled females may either be chromosomally balanced as above, or they may carry the translocated segment as a duplication in addition to two intact chromosomes of linkage group 1 ; the latter are thus aneuploid. Using $\mathrm{X}$ for a normal $\mathrm{X}$-chromosome and $\mathrm{X}^{\mathrm{T}}$ for an $\mathrm{X}$-chromosome

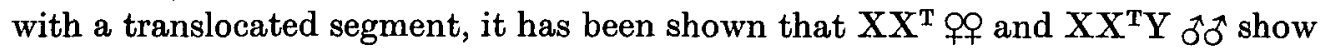
mottling, but that $\mathrm{X}^{\mathrm{T}} \mathrm{Y} \delta \sigma^{\pi}, \mathrm{X}^{\mathrm{T}} \mathrm{X}^{\mathrm{T}} \phi Q$ and $\mathrm{X}^{\mathrm{T}} \mathrm{O}$ \&ᄋ are not mottled. The mottled phenotype is thus confined to mice with one $\mathrm{X}$ and one $\mathrm{X}^{\mathrm{T}}$ (which carries the normal allele of the gene which shows mottling, such as $+^{b},+^{c},+^{p}$, etc.), but is independent of the Y-chromosome.

The mottling observed in these translocation heterozygotes has in the first instance been interpreted as a V-type position effect (Russell \& Bangham, 1961 ; Cattanach, $1961)$, and for $\mathrm{T}(X ; 8)$, at any rate, this has been conclusively demonstrated. In Drosophila (Lewis, 1950), a gene normally carried in a euchromatic region may give rise to a variegated phenotype on being transferred to the vicinity of heterochromatin. As all eight cases so far known in the mouse are $\mathrm{X}$-autosome translocations and none have been found involving two autosomes (which should be much 
commoner), it is legitimate to conclude that it is something in the $\mathrm{X}$-chromosome which communicates itself to translocated autosomal genes and thus leads to a mottled phenotype. From here it is but one step to connect the phenomenon with the L.H. by assuming that translocated segments of autosome are inactivated along with the $\mathrm{X}$-chromosome to which they have become attached; on this assumption, the mottling showing the recessive phenotype is seen in the descendants of those cells in which the $\mathrm{X}$ carrying the translocated segment of autosome (with the normal allele) has been inactivated.

This simple interpretation runs into difficulties when the behaviour of several genes carried in the same translocation, and that of several translocations involving the same pair of chromosomes is considered. As shown by Cattanach and by Russell and her collaborators, the tendency to mottling seems to spread directionally from the $\mathrm{X}$-chromosome into the translocated segment of autosome. For instance, in a female of the constitution $\mathrm{XX}^{\mathrm{T}} ; c^{c h} p / c^{c h} p$, some areas of the coat show mottling for $c^{c h}$ alone, a few for both genes, but mottling for $p$ alone does not occur : in this case, the serial order is break-ch-p. By contrast, where the order is break-p-ch, the situation is reversed. Secondly, genes nearer the break tend to show more and larger areas of mottling than genes farther away; an extreme case is $T(X ; 8)$ where $b$, some 13-14 units from the break, shows a striking degree of variegation whereas $m$, some 8 units beyond $b$, shows none at all. There is thus not only a directional 'spreading effect', but also a corresponding gradient of intensity. Thirdly, in one of the $\mathrm{X} ; 1$ translocations, $c$ shows no mottling though it is very close to the break : in this case, Russell (1963) concludes that the $c$-locus has become attached to a region of the $\mathrm{X}$ which does not lead to inactivation of attached segments of autosome and which presumably does not undergo inactivation itself. Russell $(1963,1964)$ thus postulates that not the whole $\mathrm{X}$-chromosome is liable to inactivation, but that inactivation spreads from a centre directionally and with diminishing intensity and may thus communicate itself to attached segments of autosomes (for comments see Lyon, 1966).

A mottled or variegated phenotype, in itself, does not reveal whether it is the result of events which have happened in individual cells and which have persisted during clonal growth, or whether it is the result of a widespread process which has become visible locally by a threshold mechanism. Similarly, the fact that mottling is confined to mice with both an $\mathrm{X}$ and an $\mathrm{X}^{\mathrm{T}}$ is equally compatible with the interpretation that it stems from the inactivation of either $\mathrm{X}$ or $\mathrm{X}^{\mathrm{T}}$, or that it is the result of interaction between $X$ and $X^{T}$. It thus appears that variegation in these $X$-autosome translocations, in itself, again fails to discriminate: it can be accounted for equally validly in either conceptual framework. The reason why it has been interpreted in terms of the L.H. or one of its modifications is the phenotypical similarity of mottling with heterozygotes of the mottled series $\left(M o, M o^{b r}, M o^{d p}\right.$, etc.) and the assumption that the L.H. is in agreement with the behaviour of sex-linked genes carried in normal chromosomes. As it has now been shown that there is no evidence for the inactivation of sex-linked genes in the mouse, there is no valid reason why the mottling in $\mathrm{X}$-autosome translocations should be interpreted in terms of the 
L.H. Indeed, it will be shown below that the facts are incompatible with the L.H. when considered in conjunction with the behaviour of Searle's translocation.

Quite independently of these general considerations, the mottled animals may, of course, be subjected to the various test criteria discussed in detail elsewhere (Grüneberg, 1966b). For instance, according to the L.H., the phenotype of wildtype patches should correspond to that of $+/+$ and that of mutant patches to $a / a$ (where $a$ stands for any of the 'recessives' involved); by contrast, the usual interpretation would also be compatible with intermediate phenotypes which may result when both alleles act together. So far, Cattanach's translocation only has been studied by the present author. All five females examined, in addition to black and white hairs, had numerous fibres of intermediate type, both among overhairs and in the underfur; some hairs are predominantly white, but have small amounts of pigment, others contain medium amounts of pigment; indeed, it appears that there are all intergrades between the extremes though, in near-normal hairs, a reduction in the amount of pigment is more difficult to establish critically. An ad hoc assumption to rescue the L.H. would be that many hair follicles have mixed populations of melanocytes, with varying proportions 'active' or 'inactive' respectively. However, this does not agree with the finding that pigment in intermediate hairs is commonly not uniformly spread along the shaft: pigmentation is generally heaviest near the tip and tends to diminish or vanish altogether lower down. It is difficult to see how this flagging of pigment formation during hair growth could be accounted for in terms of the L.H. The remaining $\mathrm{X}$-autosome translocations have not yet been examined, but it may be expected that they behave similarly.

Cattanach's translocation includes the normal allele of shaker-1 (sh-1), a mutant with a characteristic labyrinthine pathology. Drs M. S. Deol and M. C. Green are at present engaged in studying the behaviour of this gene in $\mathrm{XX}^{\mathrm{T}} ; s h-1 / s h-1$ co, and the results will be published in due course.

As discussed elsewhere (Grüneberg, 1966 b), according to the L.H., the manifestation of contrasted alleles in heterozygotes should be refractory to selection. 'Successful selection for or against the manifestation of alleles in heterozygotes would demonstrate genetic (Fisherian) control of dominance at the level of the gene ... Random chromosome inactivation, ex hypothesi, involves absence of any control and hence failure to respond to selection.' Cattanach \& Isaacson (1965) have succeeded by selection in establishing two strains of that translocation which differ significantly in the amount of white flecking. Whereas this could scarcely be explained in terms of the L.H., it is easy to understand that pigmentation in such $+/ c / c$ heterozygotes is influenced by the genetic background.

We mention here two experiments (Cattanach, 1963; Lyon, 1963) which were designed to test the L.H. and which have given contradictory results. Both authors produced repulsion heterozygotes in which one $\mathrm{X}$-chromosome was structurally normal and carried an allele of the mottled series; the other $\mathrm{X}$-chromosome (here symbolized as $\mathrm{X}^{\mathrm{T}}$ ) was Cattanach's translocation and thus carried the normal alleles of albinism and of pink-eyed dilution $\left(t^{c}\right.$ and $+^{p}$ respectively). Cattanach's mice were of the constitution $\mathrm{X}\left(M o^{b r}\right) / \mathrm{X}^{\mathrm{T}} ; c / c$ and 'The phenotype was a variegation for three coat colours $\left(+, c, M o^{\text {br }}\right)$ rather than two $(+, c)$, indicating that both $M o^{b r}$ on the normal X and $+^{c}$ on the rearranged X were genetically active in some cells'. Lyon's 
mice were of the constitution $\mathrm{X}\left(M o^{d p}\right) / \mathrm{X}^{\mathrm{T}} ; p / p$; as $p$ is farther away from the breakage point than $c$, mottling is far less marked on account of the 'spreading effect' and hence less conspicuous; the mice looked like ordinary $M o^{d p} /+q Q$, i.e., they showed no mottling for $p$; this was interpreted as meaning that either $\mathrm{X}\left(M o^{d_{p}}\right)$ or $\mathrm{X}^{\mathrm{T}}$ was 'active' in a given cell, but never both simultaneously.

The situation is the same as in ordinary double heterozygotes discussed in an earlier section: the validity of both experiments as tests of the L.H. rests on the assumption that the mottled alleles used (and, of course, the Cattanach translocation) individually behave in accordance with the L.H. But it is now known that in $M o^{b r} /+q Q$, at any rate, the coat as a whole is involved, and not only the light areas; the same is presumably true for $M o^{d p} /+$ of which have curly whiskers like $M o^{\text {br }} /+$ of and a positive correlation between curliness of whiskers and lightness of coat (Phillips, 1961). Hence the outcome of both experiments is the result of factor interaction and thus irrelevant to the present discussion.

\section{IS THE LYON HYPOTHESIS TENABLE?}

On the phenotypical level, the L.H. stands and falls with the behaviour of single heterozygotes of sex-linked genes carried in structurally normal chromosomes. If these single heterozygotes behave according to the postulates and consequences of the hypothesis, the L.H. survives to be further tested in more complex situations. But if the single heterozygotes behave contrary to the L.H., the complex genotypes cannot retrieve the situation: for it turns out that their interpretation is dependent on that of the behaviour of single heterozygotes and consequently non-discriminant. For instance, the phenotype of double heterozygotes is relevant for the L.H. provided the single heterozygotes are a patchwork of clonal territories; but if they are the result of threshold mechanisms and the genes act over the coat as a whole, the phenotype is the result of factor interaction and hence irrelevant in the context of this discussion. The situation is similar in the case of the $\mathrm{X}$-autosome translocations. As explained in more detail above, their interpretation is, in the first instance, dependent on that of the single heterozygotes in structurally normal chromosomes, though, of course, still subject to further tests.

By all available criteria, the behaviour of individual sex-linked genes in the mouse disagrees with the consequences of the L.H. (Grüneberg, 1966a, 1966 b). There is no evidence for gene inactivation, * but ample evidence that both $\mathrm{X}$-chromosomes are active in the same cells in $\mathrm{XX}$ 우. Hence there is no valid reason why the genetic situations discussed in this paper should be interpreted in terms of the L.H.

The observational data on double heterozygotes and $\mathrm{X}$-autosome translocations, so far as they go, are in agreement with this conclusion. For instance, there is clear evidence for factor interaction in repulsion double heterozygotes which is excluded by the L.H.; and both the phenotype (on the microscopic level) and its response to selection of one of the $\mathrm{X}$-autosome translocations points in the same

* Lyon (1966) who wrote before the publication of my $1966 b$ paper, argues that, as the inactivation of $T a$ is proved both by the phenotype of $T a /+$ and the behaviour of the gene in T16H, the discrepant behaviour of the molars of $T a /+$ of $($ Grüneberg, 1966a) must be accommodated within the framework of the L.H. by means of an ad hoc hypothesis; that offered, though rather elaborate, fails to account for the observed facts. As there is no evidence that $T a$ is, in fact, inactivated, the ad hoc hypothesis may be dispensed with. 
direction. Moreover, the 'spreading effect' and its intensity gradient cannot be accommodated in the L.H. in its original form. Indeed, the present author regards it as altogether untenable.

In this re-assessment of the basis of the L.H., attention has in the first instance been focused on the various types of genetical evidence thought to support that hypothesis. No attempt has yet been made to scrutinize the cytological basis of the L.H. Should it be found that the relevant cytological observations uniquely lead to an interpretation in terms of the L.H., we would be faced with a situation which is quite novel in the history of genetics, a contradiction between genetical and cytological evidence. However, that situation has not yet arisen.

\section{AN ALTERNATIVE HYPOTHESIS}

In view of the conclusion reached in the preceding section, I feel impelled to put forward an alternative hypothesis of gene action in the mammalian X-chromosome which is consistent with all the relevant genetical facts. It will have to reconcile the behaviour of the various $\mathrm{X}$-autosome translocations with the notion that, in the mammalian female, the genes on both $\mathrm{X}$-chromosomes are active in the same cells; and it should provide a mechanism for dosage compensation. On the other hand, it does not have to account for the phenotype of individual sex-linked genes. The phenotypical resemblance of some of the heterozygotes for sex-linked genes in the mouse is largely due to the fact that as many as six out of ten loci affect the coat. This may be an accident of sampling; but even if it were not, the bunching of genes affecting the coat could scarcely be attributed to a peculiarity of the $\mathrm{X}$-chromosome as such: more probably it would be analogous to the bunching of homoeotic mutants in the third chromosome of Drosophila melanogaster. As the phenotypes of the relevant heterozygotes are the result of threshold mechanisms rather than of clonal growth of the descendants of randomly inactivated stem cells, they do not require a special explanation any more than those of their autosomal counterparts. Whereas the phenotype of individual sex-linked genes in the mouse thus does not require a special explanation, a property of the sex-linked genes as a group possibly does. It appears that the incidence of semi-dominant genes is higher in the $\mathrm{X}$ than in the autosomes; at least six out of a total of ten loci fall into this category ( $T a, S t r, M o, G s, B n, G y$ ), and sparse-fur (spf), it seems, can also no longer be regarded as a recessive condition. By contrast, roughly three out of four autosomal loci in the mouse show no easily detectable effects in heterozygotes. This suggests 'that something in the $\mathrm{X}$-chromosome may tend to keep alleles in a state of semi-dominance rather than to allow one allele to gain complete ascendancy over the other' (Grüneberg, 1966 b). As this is clearly a moot point, a new hypothesis does not necessarily have to account for it.

To use an analogy, a cart may be pulled by one horse. If it is pulled by a team of two horses, each of them will pull with approximately half-strength. This is the hypothesis which will be developed here. By contrast, the L.H. postulated that; 
in the case of the two-horse cart, one of the horses is unhitched and led by the bridle so that, in effect, the cart is always pulled by one horse working at full strength.

The new hypothesis incorporates Russell and Cattanach's 'centre of inactivation' though with a different interpretation. Thus there exists a 'centre' in the mammalian $\mathrm{X}$-chromosome from which an inhibition of gene action spreads along the $\mathrm{X}$ and, with diminishing intensity, into attached segments of autosome. This 'centre' is activated only if there are two $X$-chromosomes present (as shown by the fact that, in the $\mathbf{T}(X ; 1)$ and $T(X ; 8)$ translocations, mottling is confined to $\mathrm{XX}^{\mathrm{T}}$ or? and

\section{$X$-AUTOSOME TRANSLOCATIONS}

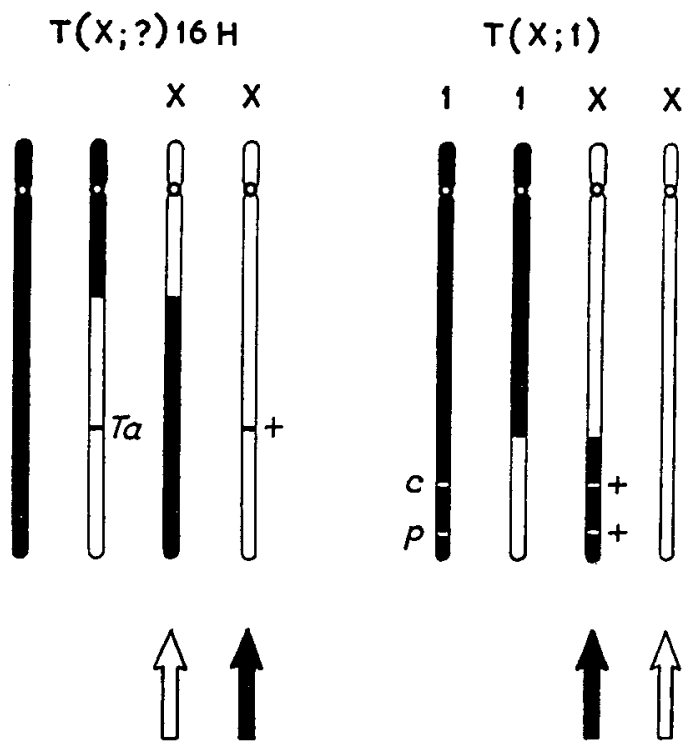

Fig. 1. The X-autosome paradox. In each type of translocation, one X-chromosome is manifestly involved (black arrows): in Searle's translocation $(\mathrm{T}(\mathrm{X} ;$ ? $) 16 \mathrm{H})$, the intact $\mathrm{X}$-chromosome; in the $\mathrm{X} ; \mathrm{l}$ and $\mathrm{X} ; 8$ translocations, the $\mathrm{X}$-chromosome with the translocated segment of autosome. The paradox is resolved by the assumption that in both instances, the other X-chromosome is similarly involved (white arrows) though this is not detectable in the absence of suitable marker genes.

$\left.\mathrm{XX}^{\mathrm{T}} \mathrm{Y} \circlearrowleft \widehat{\delta}\right)$. The essential new postulate of the hypothesis is that, in the mammalian female, both centres are activated together and that the inhibiting action spreads along both $\mathrm{X}$-chromosomes.

The observational basis for this new postulate is a paradox (Fig. 1) which seems to have escaped notice or, at least, discussion. The paradox is equally at variance with the L.H. in its original form and with its modification due to Russell and to Cattanach. In Searle's translocation, whichever sex-linked allele is removed from the $\mathrm{X}$-chromosome becomes dominant over the allele on the intact $\mathrm{X}$ : inhibition thus happens in the intact $\mathrm{X}$-chromosome. By contrast, in the $\mathrm{T}(\mathrm{X} ; 1)$ and $\mathrm{T}(\mathrm{X} ; 8)$ translocations, inhibition involves the autosomal genes attached to the $\mathrm{X}$ chromosome, i.e., it originates in the $\mathrm{X}$-chromosome carrying the translocation. This 
paradox disappears if it is postulated that, in either type of translocation, inhibition involves both $\mathbf{X}$-chromosomes though, in each instance, it is detectable only in one. As in either type of translocation heterozygote one of the $\mathrm{X}$-chromosomes is structurally normal, it must be concluded that a similar inhibition will also affect both structurally normal $\mathrm{X}$-chromosomes in normal mouse females. Whereas individually, both types of $\mathrm{X}$-autosome translocation were non-discriminant as regards the L.H., when they are considered in conjunction, they cannot be accounted for on the assumption that a single $\mathrm{X}$-chromosome is inactivated (as postulated by the L.H.). But if both $\mathrm{X}$-chromosomes are involved, inhibition of gene action cannot be complete: it must be incomplete, i.e., the two alleles in heterozygotes act together like autosomal genes, a conclusion reached quite independently from the analysis of their phenotypic effects (Grüneberg, 1966a, 1966b).

As discussed in a preceding section, the behaviour of the genes in Searle's translocation, viewed in isolation, could also be formulated as due to an enhancement of the action of the sex-linked genes beyond the normal when translocated to an autosome: nothing actually happening in the intact $X$. But the behaviour of the autosomal genes in the $T(X ; 1)$ and $T(X ; 8)$ translocations cannot be formulated without ascribing the effect to something in the X-chromosome. As we cannot arbitrarily invoke different mechanisms for the two types of translocation, we have to adopt the interpretation which fits them both; i.e., that in both instances, the observed effects originate in the X-chromosome.

The new hypothesis is based on the behaviour of sex-linked genes in ordinary heterozygotes and on that of both sex-linked and autosomal genes in $\mathrm{X}$-autosome translocations. It is consistent with the whole of this evidence.

(1) In the normal female, genes on both $\mathrm{X}$-chromosomes are active in the same cells, as established for $T a, M o^{b r}$, Str and probably for $B n$.

(2) In Searle's translocation, normally $\mathrm{X}$-borne genes, on being translocated to an autosome, escape from the effect of the inhibiting centre and thus become dominant over their alleles on the intact $X$ which remain exposed to partial inhibition.

(3) In the $T(X ; 1)$ and $T(X ; 8)$ translocations, autosomal genes translocated to the $\mathrm{X}$ may come under the influence of the centre and thus be partially inhibited; the magnitude of the effect being subject to distance from the breakage point.

(4) If indeed the high incidence of semi-dominant sex-linked genes in the mouse should require a special explanation, it may be connected with the postulate that the two alleles act at about half-strength each (though the exact mechanism is not clear).

The postulate that, in the mammalian female, two sex-linked alleles jointly do the work of one, of course provides a basis for dosage compensation in much the same way as does the L.H. In the absence of factual information, speculations on the physiological (molecular) mechanism(s) which may bring this about would be of little value.

In conclusion, it is perhaps worth pointing out that the critical attitude towards the L.H. in this series of papers is not meant to imply that it lacks scientific merit: 
it has focused interest on a problem which, in mammals at any rate, had previously received scant attention. It is also a good hypothesis in the sense that it is open to verification or falsification in many different ways. The present author is under no illusion that his own hypothesis, like the L.H., will eventually be replaced by a yet better approximation to the truth.

\section{SUMMARY}

Contrary to opinions expressed by various authors, the phenotype of heterozygotes for mammalian sex-linked genes gives no support for the Lyon hypothesis (L.H.). Evidence, mainly from the mouse, shows that in such heterozygotes, both alleles act together as in autosomal genes.

In the present paper, it is shown that neither the behaviour of double heterozygotes for sex-linked genes nor that of $\mathrm{X}$-autosome translocations provides independent evidence in favour of the L.H. : in each case, the interpretation depends on that of the behaviour of single heterozygotes and hence fails to discriminate. Moreover, new facts from both types of situation are also contrary to the L.H. In particular, a unified interpretation which fits the behaviour of genes in all known types of $\mathrm{X}$-autosome translocations in the mouse requires the assumption that partial inhibition of gene action happens in both $X$-chromosomes of mouse females, and presumably the females of other mammals. The new hypothesis is consistent with all relevant genetical facts and, like the L.H., it also accounts for dosage compensation.

The author is indebted to Drs D. S. Falconer, Mary F. Lyon and B. M. Cattanach for the gift of several mouse mutants and translocation stocks, and to Dr Gillian M. Truslove and Miss Jean M. Gray for assistance in various ways.

\section{REFERENCES}

Cattanach, B. M. (1961). A chemically-induced variegated-type position effect in the mouse. $Z$. VererbLehre, $92,165-182$.

Cattanach, B. M. (1963). The inactive- $X$ hypothesis and position effects in the mouse. Genetics, 48, 884-885 (Abstr.).

Cattanach, B. M. (1966). The location of Cattanach's translocation in the X-chromosome linkage of the mouse. Genet. Res. 8, 253-256.

Cattanach, B. M. \& IsaAcson, J. H. (1965). Genetic control over the inactivation of autosomal genes attached to the X-chromosome. Z. VererbLehre, 96, 313-323.

Falconer, D. S., Fraser, A. S. \& King, J. W. B. (1951). The genetics and development of 'crinkled', a new mutant in the house mouse. J. Genet. 50, 324-344.

GRÜNEBERG, H. (1965). Genes and genotypes affecting the teeth of the mouse. J. Embryol. exp. Morph. 14, 137-159.

GrüneBERG, H. (1966a). The molars of the tabby mouse, and a test of the 'single-active X-chromosome' hypothesis. J. Embryol. exp. Morph. 15, 223-244.

GRÜNEBERG, H. (1966b). More about the tabby mouse and about the Lyon hypothesis. J. Embryol. exp. Morph. 16, 569-590.

GrüneberG, H. (1967). Sex-linked genes in man and the Lyon hypothesis. Ann. hum. Genet. 30, 239-257.

Lewis, E. B. (1950). The phenomenon of position effect. Adv. Genet. 3, 73-115. 
Lyon, M. F. (1961). Gene action in the X-chromosome of the mouse (Mus musculus L.). Nature, Lond. 190, 372-373.

Lyon, M. F. (1962). Sex chromatin and gene action in the mammalian X-chromosome. Am. J. hum. Genet. 14, 135-148.

Lyon, M. F. (1963). Attempts to test the inactive-X theory of dosage compensation in mammals. Genet. Res. 4, 93-103.

Lyon, M. F. (1966). Lack of evidence that inactivation of the mouse X-chromosome is incomplete. Genet. Res. 8, 197-203.

Lyon, M. F., Searle, A. G., Ford, C. E. \& Ohno, S. (1964). A mouse translocation suppressing sex-linked variegation. Cytogenetics, 3, 306-323.

Ohno, S. \& Cattanach, B. M. (1962). Cytological study of an X-autosome translocation in Mus musculus. Cytogenetics, 1, 129-140.

OHNo, S. \& LyoN, M. F. (1965). Cytological study of Searle's X-autosome translocation in Mus musculus. Chromosoma, 16, 90-100.

Phillirs, R. J. S. (1961). 'Dappled', a new allele at the mottled locus in the house mouse. Genet. Res. 2, 290-295.

RUSSELL, L. B. (1963). Mammalian X-chromosome action : inactivation limited in spread and in region of origin. Science, N.Y. 140, 976-978.

Russeld, L. B. (1964). Another look at the single-active-X hypothesis. Trans. N.Y. Acad. Sci., ser. II, 26, 726-736.

Russeli, L. B. \& Bangham, J. W. (1961). Variegated-type position effects in the mouse. Genetics, 46, 509-525.

Russell, L. B., Bangham, J. W. \& Montgomery, C. S. (1964). The use of X-autosome translocations in the mouse for the study of autosomal genes. Genetics, 50, 281-282 (Abstr.).

Russell, L. B., Bangham, J. W. \& Saylors, C. L. (1962). Delimitation of chromosomal regions involved in V-type position effects from $\mathrm{X}$-autosome translocations in the mouse. Genetics, 47, 981-982 (Abstr.).

Russelt, L. B. \& Montaomery, C. S. (1965). The use of X-autosome translocations in locating the $\mathrm{X}$-chromosome inactivation center. Genetics, 52, 470-471 (Abstr.). 